\title{
Du meurtre symbolique du père
}

Et de l'aspect insaisissable du présent

About the Symbolic Murder of the Father and the Elusive Aspect of the Present 象征性斌父與當下游離

\section{Brigitte Baptandier}

\section{(2) OpenEdition \\ Journals}

Édition électronique

URL : http://journals.openedition.org/extremeorient/237

DOI : 10.4000/extremeorient.237

ISSN : 2108-7105

\section{Éditeur}

Presses universitaires de Vincennes

\section{Édition imprimée}

Date de publication : 1 janvier 2012

Pagination : 277-312

ISSN : 0754-5010

\section{Référence électronique}

Brigitte Baptandier, «Du meurtre symbolique du père ", Extrême-Orient Extrême-Occident [En ligne], Hors-série | 2012, mis en ligne le 01 janvier 2015, consulté le 30 avril 2019. URL : http:// journals.openedition.org/extremeorient/237 ; DOI : 10.4000/extremeorient.237 


\title{
Du meurtre symbolique du père et de l'aspect insaisissable du présent
}

\author{
Brigitte Baptandier
}

«Le symbolique, l’imaginaire et le réel, voilà les vrais noms du père.»

«"Le Nom-du-Père, dit Lacan, crée la fonction du père." Mais alors, ce pluriel, d'où vient-il ? [...] Il n'est pas païen, il est dans la Bible. Celui qui parle dans le buisson ardent dit de lui-même qu'Il n'a pas qu'un seul Nom. Entendons: le Père n'a pas de Nom propre. Ce n'est pas une figure, c'est une fonction. Le Père a autant de Noms qu'elle a de supports. Sa fonction? La fonction religieuse par excellence, celle de lier. Quoi? Le signifiant et le signifié, la Loi et le désir, la pensée et le corps. Bref, le symbolique et l'imaginaire. Seulement, si ces deux se nouent à trois avec le réel, le Nom-du-Père n'est plus qu'un semblant. En revanche, si sans lui tout se défait, il est le symptôme du nœud raté.»

Jacques Lacan, Des Noms-du-Père, préface, p. 7, et quatrième de couverture.

Avant toute chose, il nous faudra examiner, rapidement, quelques paradoxes structurels issus des règles canoniques anciennes de la Chine quant à la parenté et au deuil. Ils nous permettront d'interroger la place du père dans l'espace (rituel) des générations, telle qu'elle est conçue, aujourd'hui encore, dans le contexte de la société moderne. Nous observerons la place du nom du père dans le tissage du masculin (la chaîne des générations, verticale) et du féminin (la trame, horizontale), quant à l'alliance et à la procréation. Nous envisagerons le partage de l'espace (maison, lignage) entre les genres. Nous exposerons alors les catégories de sexe et les fantasmes issus de la crainte qu'un réseau féminin antigénéalogique (rhizome) ne mette en danger l'arborescence patrilinéaire (lignée), prédominante dans les règles de parenté chinoise. Dans un deuxième temps, nous aborderons 
le thème du destin d'un enfant - garçon ou fille - mettant son père en danger de par la position astrologique du temps de sa naissance. Nous en viendrons aux procédés de sublimation de telles situations et aux « remèdes empoisonnés » qui leur sont trouvés. Nous verrons comment il s'agit en réalité de la crainte de l'inceste. Nous examinerons le cas, vécu comme une mutilation, du fils donné en gendre à un autre lignage, mais aussi de la fille «née épouse», afin d'éviter l'antagonisme mortel de son horoscope au destin paternel.

Finalement nous conclurons sur la génération actuelle de ces jeunes époux placés face à la situation nouvelle qui leur est imposée de n'engendrer qu'un seul enfant pour assumer l'ensemble de ces situations dans le contexte moderne et l'obligation qui leur est faite, en dépit de tout, de prendre en charge l'ancestralité. Nous serons amenés à considérer la place cruciale des générations alternées dans cette parenté réinventée et dans un contexte social où leur importance aurait fort bien pu disparaître.

\section{De quelques paradoxes canoniques et de leurs conséquences}

\section{De la piété filiale: le père est un fils}

Dans la société patrilinéaire chinoise, un homme est avant toute chose un «fils» (erzi). Bien plus, il demeure de fait un «enfant» (haizi), tant qu'il n'a pas produit lui-même un rejeton mâle: dès lors, il prend la place de père (fuqin), statut qui ne lui est reconnu qu'au titre d'instituer son propre père en ancêtre $(z u)$ grâce à cette prolongation de la lignée nécessaire pour perpétuer le culte ancestral. En somme, le père représente l'aspect insaisissable du présent. Comme le dit Françoise Lauwaert:

Le père est chassé de sa position centrale pour se trouver engagé dans un processus de translation tout au long des générations. Il ne tire pas sa légitimité de son rôle de chef de famille, mais bien de son statut de fils, de père et de futur ancêtre. Il est pris dans le flux du temps. Son talent, sa personnalité, sont de peu d'importance, seules entrent en ligne de compte sa naissance dans telle ou telle génération et son appartenance à une lignée particulière. Il tire sa justification de sa place dans la communauté agnatique dont c'est pour lui le plus important devoir religieux que d'assurer la perpétuité: «Les rites exigent que le successeur se conduise comme un fils, et cette règle s'impose à tout le monde, de l'empereur au dernier de ses sujets ${ }^{1}$. »

Aujourd'hui encore, on entend couramment désigner, de façon parfois troublante, sous l'appellation de xiaohai, «enfant», un jeune homme pourtant déjà marié. C'est simplement dire qu'il n'est pas encore «père».

1. Lauwaert (1991):173, citant l'Histoire des Ming (Ming shi): 5056. 
Du meurtre symbolique du père et de l'aspect insaisissable du présent

\section{Des générations alternées : le père est un chef, le grand-père un parent}

Françoise Lauwaert remarque en conséquence:

Pour qu'un tel système fonctionne, il faut qu'à chaque génération s'opère avec un maximum de clarté la distribution - pour reprendre les termes de Léon Vandermeersch - des individus en lignées. C'est là qu'intervient un principe de classification intitulé l'ordre zhaomu. À l'origine ce terme désignait les places attribuées aux tablettes des descendants de l'ancêtre fondateur du lignage, qui étaient rangées dans les temples en deux séries, selon un strict principe d'alternance: le zhao correspondant sur le plan symbolique à la génération du père, et le $m u$ à celle du fils. Un individu de la catégorie zhao ne pouvait être continué que par un individu de la catégorie $m u$, et vice versa ${ }^{2}$.

Ce serait donc la théorie du deuil qui rendrait compte de la rigoureuse stratification en générations, caractéristique du système de parenté (et non l'inverse), comme le fait remarquer Lévi-Strauss à la suite de Feng Hanyi, tous deux discutant la théorie de Granet à ce sujet ${ }^{3}$. Selon le principe des générations alternées, dans le deuil le petit-fils est considéré comme le «représentant» (daibiao) du grand-père. Il pouvait même, lors des rites funéraires antiques, être nommé «cadavre» $(s h i)$ à cause de ce rôle rituel de substitut de son aïeul. C'est ce que souligne Granet, citant le Traité des rites (Liji, environ III'-II' siècle av. J.-C.) :

«Un honnête homme porte dans ses bras son petit-fils, il ne peut pas porter son fils.» La règle est interprétée comme étant imposée par le respect filial. Mais la phrase est suivie dans le texte par celle-ci: «Ceci veut dire que (dans les cérémonies du culte ancestral) le petit-fils peut servir de représentant de son défunt grand-père: un fils ne peut pas être le représentant de son père défunt ». La glose ajoute : «le petit-fils et le grand-père sont tous deux d'un même côté de la parenté». Bon exemple de règle morale dérivée d'une règle proprement rituelle. L'interdiction de porter son fils dans les bras s'entend surtout pour le temps du sacrifice: un jeune enfant qui représenterait son grand-père ne pourrait être porté que par quelqu'un qui fût du même côté de la parenté. Mais cette règle, qui marque l'éloignement de deux générations agnatiques consécutives, implique la pratique, dans la vie courante, de quantités de règles de détail où se manifeste cet éloignement ${ }^{4}$.

Cette opposition entre deux générations consécutives - celle du père et celle du fils - amène les canons rituels antiques à envisager des situations de distance extrême entre elles. Comme le dit encore Granet pour cette période lointaine des Hégémons qu'il étudie:

2. Lauwaert (1991): 7, se référant à Léon Vandermersch (1977-1980), I: 239-260.

3. Voir Lévi-Strauss (1967), «L'ordre Tchao-Mou (zhaomu): 384, et Feng Hanyi (1937).

4. Granet (1959): 274, note 2, citant le Liji, traduction Couvreur, t. 1: 47. 
Le père ne peut porter son fils dans ses bras; le fils ne peut habiter où demeure son père; dans le temple ancestral, père et fils sont logés, non point côte à côte, mais face à face; les tablettes représentant les ancêtres de deux générations consécutives ne peuvent être rangées d'un même côté du bâtiment; jamais, même quand tous deux sont morts, le fils ne mange avec son père. Bien que la filiation soit agnatique et que la succession se fasse de père à fils, le gouvernement de la famille appartient alternativement à deux groupes opposés ${ }^{5}$.

[...] Tandis que la Terre et la Mère sont prochaines et parentes, le Père, comme le Ciel, est majestueux et sévère. L'analyse des rapports entre père et fils montre qu'il y a entre eux un éloignement que cherchent à atténuer peu à peu des rites d'approche, mais qui reste toujours extrême. Le père reste pour le fils autre chose qu'un parent: c'est un chef. Le fils s'inféode à lui, il ne réussit pas à s'apparenter à lui. Le grandpère, au contraire, est un proche, un parent ${ }^{6}$.

Pourtant, Lévi-Strauss regrettait, par ailleurs, que dans son commentaire de la théorie de Granet sur ce sujet, Francis Hsü, spécialiste de l'étude des lignages de la Chine du Sud, n'ait pas analysé le système zhaomu à la lumière de l'ethnographie des coutumes modernes du Fujian, avant de déclarer que l'ordre zhaomu n'avait rien à voir avec une ancienne structure de parenté, et que, tout simplement: "l'ordre tchao mou (zhaomu) ne s'est jamais rapporté qu'à la relation entre pères et fils ${ }^{7} \gg$. Lévi-Strauss remarque à ce sujet, en conclusion de son chapitre: «Le principe des générations alternées a pris, au cours de ces dernières années, une telle importance dans l'interprétation de phénomènes jusqu'alors obscurs, que partout où l'on trouve une ébauche, ou un vestige, d'opposition entre générations consécutives et d'identification de générations éloignées, un préjugé favorable doit intervenir ${ }^{8}$.»

5. Granet (1959): 274.

6. Granet (1959): 274, note 1 .

7. «Peut-être l'ethnographie pourrait-elle éclairer le problème par l'observation de coutumes modernes? Ici encore, on déplorera que Hsu ait rejeté sans examen une suggestion de Radcliffe Brown, l'invitant à étudier l'ordre tchao mou (zhaomu) à la lumière des usages du Fou Kien (Fujian) où, aujourd'hui encore, le mariage avec la cousine patrilatérale est strictement interdit et où les ancêtres sont toujours placés alternativement à droite et à gauche du fondateur. On a du mal à admettre $a$ priori qu'il n'y ait aucun rapport entre ces usages et le rituel Chou, tel que l'évoque Maspero [...]». In Lévi Strauss (1967): 396. Voir aussi Hsü (1940): 257-259.

8. Lévi-Strauss (1967): 96, citant Maspero (1927): 251-252 à propos du dispositif funéraire, c'est-à-dire l'emplacement des tablettes dans les temples, ajoute: «Le premier ancêtre occupait le haut bout, face à l'est, son fils à sa gauche, face au sud, son petit-fils à sa droite, face au nord, et tous les descendants rangés ainsi en deux files, les tchao (zhao) à la gauche, les mou $(\mathrm{mu})$ à la droite à la suite du fils et du petit-fils respectivement, s'éloignant de plus en plus de l'ancêtre à chaque génération... Tous les mou $(m u)$ descendants du 
Du meurtre symbolique du père et de l'aspect insaisissable du présent

Nous verrons d'ailleurs comment les générations alternées trouvent dans la société actuelle, naturellement fort différente, une place particulièrement déterminante en regard de la règle, imposée désormais, de l'enfant unique.

\section{Du partage de l'espace: la maison et le lignage}

Dans son ouvrage sur la construction de l'espace en Chine ancienne (notamment au moment de l'unification de l'empire Han), Lewis s'intéresse au modèle de construction de la société humaine telle qu'elle est présentée à travers la mise en forme progressive et la distribution dans l'espace des objets et des groupes ${ }^{9}$. Virtuellement, toutes les théories chinoises sur la société et le monde physique partent, selon lui, de la présomption d'un chaos (luan) originel indifférencié, demeurant en arrière-plan à la fois comme la source d'un pouvoir sous-jacent et comme la menace de graves conséquences en cas d'actions inappropriées induisant le risque de plonger à nouveau dans cette indistinction. Cette idée d'un état de confusion primordiale, à la fois source de vitalité et menace de chaos destructeur, reste, d'après Lewis, un trait de la pensée chinoise jusqu'à aujourd'hui ${ }^{10}$.

La création d'un espace structuré à partir d'un chaos est également présente dans les récits sur un âge présocial où les hommes et les animaux vivaient librement ensemble ${ }^{11}$. Ces récits insistent sur l'importance de continuer à agir, dans le temps qui leur est contemporain, pour protéger cet espace de la menace du retour au chaos. Les discussions à propos du rituel assurent d'ailleurs qu'il maintient l'ordre en imposant des divisions ${ }^{12}$. C'est en séparant les hommes des femmes, les aînés des plus jeunes, les dirigeants de leurs sujets ou les civilisés des barbares que le rituel a distribué les rôles et constitué les groupes sociaux.

L'univers de la maison ou de la famille (jia) en tant qu'unité spatiale englobant les corps, envisagée en référence à des unités plus larges qui l'englobaient et dont elle dépendait - telles que l'État ou le groupe de parenté formé par le lignage $(z u)$ - relevait de ce modèle. La maison elle-même était présentée comme une unité divisée, en son propre sein, entre les genres. La division entre les hommes et les femmes y était perceptible tant sur le plan économique - l'agriculture était

roi Wen sur une ligne à sa gauche, tous les tchao (zhao) descendants du roi Wou $(W u)$ sur une ligne à sa droite, et tous les тои $(\mathrm{mu})$ et les tchao (zhao) antérieurs à ces deux rois... en deux rangées à gauche et à droite du premier ancêtre Heou-tsi (Hou Ji)».

9. Lewis (2006).

10. Lewis renvoie à Denton (1998): 222-243, au sujet de cette vision parallèle appliquée à l'état sauvage primordial dans le roman ( $\mathrm{xx}^{\mathrm{e}}$ siècle) de Lu Ling: Caizhu de ernümen (Enfants de riches).

11. Voir Lewis (1990): 165-174 et Lewis (1999): 127-129.

12. Pour des exemples à ce sujet: voir Lewis (2006): chap. 2, note 142. 
dévolue aux hommes et la production de vêtements aux femmes - que, d'un point de vue social, sur le plan de la parenté. Le lien majeur y était, en effet, celui entre le mari et l'épouse, alors qu'au sein du patrilignage prévalait le lien entre le père et le fils, ou plus exactement entre les hommes appartenant à différentes générations. Les tensions entre ces deux groupes de parents s'exprimaient notamment à travers deux types d'attitudes contradictoires adoptées à l'égard des femmes au sujet de leur place dans la maison. D'une part les femmes exerçaient un pouvoir réel au sein de la maisonnée (le pouvoir des mères sur les fils en atteste), d'autre part la littérature canonique refusait de sanctionner ce pouvoir. Ce paradoxe transparaissait également dans la logique spatiale: l'intérieur des maisons ou des palais était tout à la fois le locus de la plus haute autorité et le royaume des femmes, même si toute autorité leur était déniée.

Selon Lewis, ce partage de la maison entre les genres s'exprima de manière significative dans le culte funéraire des Han, tandis que les tombes façonnées sur le modèle des maisons devenaient un lieu majeur d'offrandes aux morts. La tombe, en tant que réplique de la maison, était partagée par le mari et son épouse. En tant que telle, la femme y avait un rôle dominant, alors que dans le temple dévolu à la structure masculine, les femmes n'étaient que de simples compléments. Ces deux types de cultes funéraires démontrent l'importance des tensions à la fois entre le lignage et la maison mais aussi entre les sexes.

\section{De la rivalité entre les sexes : la fête des relevailles}

Malgré cette distance imposée, dans l'Antiquité comme de nos jours, un père reconnaît traditionnellement son enfant, fils ou fille, en lui donnant le nom du lignage de ses ancêtres, xing, et en lui attribuant un nom personnel, ming, lors de la fête des relevailles, dite rite du «mois plein» (manyue). À ce moment-là, en le «faisant rire», il lui transmet cette parcelle du souffle commun au lignage qui l'anime désormais.

Granet décrit cette cérémonie de la sorte, d'après le Liji:

Au troisième mois, l'enfant pris sur les bras par la mère, avait été présenté au père (il savait alors regarder et était capable de rire): le père le flattant de la main et le faisant rire, lui donnant, avec un nom personnel, une personnalité et l'intelligence, avait ordonné qu'on prît soin de l'élever: il était alors devenu quelqu'un dont on ne pourrait certes pas déjà porter le deuil, mais à la mort de qui il serait permis de pleurer; ses cheveux avaient été coupés pour la première fois et arrangés de manière à montrer symboliquement qu'il deviendrait un fils pieux; il avait reçu de son père la paumée et avait ainsi commencé à lui être affilié; sa mère avait pu le porter sans que pour cela il ait continué d'empêcher les rapports entre son mari et elle: tous deux, 
après un repas analogue à celui de leur mariage, avaient repris commerce ensemble. La cérémonie avait mis fin à la fois à l'isolement de la mère et à celui de l'enfant ${ }^{13}$.

Toutes choses égales par ailleurs, cette description est encore valable de nos jours et correspond à ce que j'ai pu observer à Tainan en 1980. Ce jour-là, on dresse devant l'autel familial une table d'offrandes sur laquelle on dispose des présents de nourriture. L'enfant, après avoir été baigné, est confié à sa grandmère maternelle, waipo, venue tout exprès en visite. Prenant l'enfant sur ses genoux, elle rase ses cheveux à l'endroit de la fontanelle, accomplissant ainsi le rite purificateur décisif qui marque sa séparation d'avec les forces terrestres du temps intra-utérin. L'enfant étant ainsi préparé, on met dans sa main droite trois bâtons d'encens et on le fait saluer les tablettes des ancêtres paternels et les divinités de l'autel familial : il entre définitivement dans le patrilignage. On le présente alors à son père et à son grand-père paternel, éventuellement aux oncles paternels présents. Le père prend l'enfant dans ses bras et le nomme. Le frère cadet du père, shushu, fait don à l'enfant d'un présent d'argent dans une «enveloppe rouge», hongbao. La grand-mère maternelle offre également plusieurs présents rituels : premier bonnet, premières chaussures ainsi que des offrandes symboliques telles que des cannes à sucre, parce que poussant très haut et très droit elles inspireront la même conduite à l'enfant. Elle offre également un coq pour qu'il lui montre le chemin, ou peut-être parce que le nom de cet oiseau, $j i$, est homophone de ji, bénéfique, ou encore parce que le coq est l'animal de l'aube, tout comme l'enfant se trouve à celle de sa vie. C'est aussi le nom d'une constellation et l'animal représentant l'un des douze rameaux terrestres du Taisui, la «Grande Année », astrologiquement parlant le temps yin de l'année ${ }^{14}$. On coiffe l'enfant de son premier bonnet et, s'il s'agit d'une fille, on peut l'orner d'une épingle sur laquelle sont piquées douze fleurs représentant les douze mois de l'année, chacune caractérisant un type de destin féminin. On souhaite de la sorte qu'elle avance avec bonheur dans les cycles de sa vie de femme. Ces cérémonies une fois terminées, les parents vont annoncer officiellement la naissance à tous les voisins et amis en apportant des présents de nourriture en échange desquels ils reçoivent également des cadeaux, sem-

13. Granet (1953): 185, d'après le Liji, trad. Couvreur (1950), I : 665-672.

14. Il s'agit du cycle de soixante ans (cycle de Jupiter) qui correspond à la réalisation complète de la combinaison des troncs célestes et des rameaux terrestres. Dans la représentation de la «bureaucratie céleste», Taisui est le président du ministère du Temps. Cf. Doré (1914): X, 142 sq. Les légendes de Taisui sous son aspect humain de Nazha Santaizi sont l'étoffe du Roman de l'investiture des dieux (Fengshen yanyi, par Xu Zhonglin). Pour la relation entre ces légendes, la piété filiale et la subjectivité masculine en regard des générations, voir Sangren (1996). À propos de Taisui comme étoile maléfique, voir Hou (1979). 
blables le plus souvent. Ces échanges de présents n'avaient lieu autrefois que lorsque le nouveau-né était un garçon. S'il s'agissait d'une fille, on se contentait d'annoncer sa naissance aux amis sans cérémonie particulière. Cette différence est observée moins strictement de nos jours, et pour cause, et chacun est libre de son attitude. Il est temps alors d'aller présenter l'enfant aux divinités. Cette présentation au temple ne nécessite ni rituel, ni la présence d'un officiant. On apporte simplement de nombreux fruits et une grande quantité de monnaies d'offrande et d'encens.

On voit bien à cette occasion le jeu des deux lignées en présence, paternelle et maternelle, et plus particulièrement encore celui des deux univers, presque des deux corps, masculin et féminin, du père et de la mère. Le rite de manyue, fin de l'isolement de la mère, correspondant à la reprise des relations sexuelles entre les conjoints, été célébré par un repas analogue à celui du mariage. Cette fête rituelle est toujours envisagée comme marquant la fin du temps de l'accouchement.

\section{Aux limites de l'humain : l'univers terrestre maternel}

Un pas plus loin, il existe des cas où l'enfant, n'ayant pas d' «affinité astrologique» (meiyou yuanfen) avec la famille maternelle, ne pourra pas, provisoirement, recevoir la visite de sa waipo. On dit qu'il porte la «passe des Cent Jours » (bairi guan) dans son destin. En effet, autrefois, la dation du nom personnel de l'enfant avait lieu au troisième mois. Toutefois, passé ce délai, l'enfant officiellement reconnu et nommé par son père peut sans crainte recevoir la visite de la famille maternelle. Cette absence «d'affinité » n'a, bien sûr, rien à voir avec les sentiments familiaux, mais concerne l'incompatibilité, passagère dans ce cas, des caractères de naissance de l'enfant et de ceux de la grand-mère maternelle. La waipo guan, bien significative des relations existantes avec la «famille étrangère» (waijia), celle de la mère, semble trahir une certaine difficulté de l'enfant à échapper à l'emprise de l'univers maternel, utérin. L'enfant porteur de guan est littéralement absorbé par cet univers. Son humanité même n'est pas encore bien établie. Le rituel d'ouverture des guan parle d'ailleurs constamment de l'enfant comme d'un être mi-animal mi-humain ${ }^{15}$. Il s'agit là d'un trait caractéristique de l'état de symbiose maternelle lors de la petite enfance. Il va donc falloir «désensauvager» l'enfant. Bien sûr, la finalité immédiate sera de l'inscrire fermement dans son patrilignage.

15. À propos du rituel d' «ouverture des guan», voir Berthier (1988) et Baptandier (1996a et 2008). Voir aussi Turner (1969) qui l'évoque et Huang Youde (1967). 
Du meurtre symbolique du père et de l'aspect insaisissable du présent

\section{Le nommé et le «sans-nom»}

Pour nommer un enfant, certaines familles disposent d'un «poème de lignage », jiashi. En effet, les noms personnels, ming, s'y succèdent au sein des vers et marquent la place de chacun dans les générations : chaque caractère du texte est un nom potentiel et le nom personnel du fils suivra celui du père dans le poème ${ }^{16}$. Ces familles ont alors parfois un registre lignager (jiapu) dans lequel on consigne soigneusement la succession des générations depuis l'ancêtre originel. Or, il s'agit là d'un tissage purement masculin, une chaîne sans trame, en somme. En effet, en ce qui concerne les filles, leur nom personnel n'est pas censé être pris dans le poème. Elles ne figurent pas non plus à part entière dans le registre lignager, sauf à disposer d'un caractère particulier ou à partager celui d'un homme de la famille ${ }^{17}$. Si, plus tard, elles figurent bien dans le registre lignager de leur époux, c'est uniquement sous le nom de leur lignage, xing, celui de leur père, et en tant qu'épouse du lignage de leur mari, à telle génération: «(xing), épouse de». Rubie Watson, parlant de la région de Canton, dit que les femmes perdent leur nom personnel (ming) lorsqu'elles se marient. Si ce nom leur est, de fait, utile à ce moment précis pour examiner la correspondance de leur horoscope avec celui de leur futur époux, elles ne sont ensuite plus nommées et elles ne nomment pas non plus. En ce sens elles n'atteindraient pas un «full personhood», pour reprendre l'expression de Clifford Geertz: elles demeureraient des «anybodies» et n'accéderaient pas au statut de «somebodies ${ }^{18}$. On ne s'adresse à elles que par un terme de parenté: «mère de», «épouse de». Elles sont des étrangères, des alliées, témoins du tissage des lignages dans l'alliance et garantes de l'absence de relations incestueuses par le nom, car on ne peut épouser une femme de même patronyme. En effet, le xing est de même nature que l'essence lignagère: os, sperme et riz. Il s'agirait donc d'un inceste.

16. Il s'agit des lignages ayant acquis un statut important soit par leur distinction intellectuelle, soit par leur richesse ou par leur importance locale, notamment par une charge officielle. C'est donc la tradition confucéenne qui est ici poursuivie. Cette coutume est également adoptée par certaines lignées taoïstes, le Quanzhen notamment, bien que les religieux y soient dits «sortis de la famille», chujia, pour entrer en religion. Ce poème renvoie alors non pas à un père, mais bien à un maître. Voir Herrou (2001).

17. Voir Alleton (1993): chap. 4.

18. Watson (1986): 619; Geertz (1973): 363. Cette affirmation de Rubie Watson renvoie à sa propre observation dans la région de Canton. Même s'il faut se garder de généraliser, force est de constater qu'on pourrait dire sensiblement la même chose pour d'autres régions du Sud de la Chine, le Fujian notamment. 


\section{Du nom du père et de la «flagornerie»}

Il en est de même pour le culte aux ancêtres. Les femmes ont certes un rôle dans le deuil porté pour les parents du mari, et elles ont généralement la charge quotidienne de mettre de l'encens et des offrandes sur l'autel familial. Mais ce sont les hommes qui rendent le culte, à proprement parler, à leurs ancêtres. Car seuls des hommes portant le même patronyme peuvent rendre le culte à des ancêtres communs. Comme le relève Françoise Lauwaert: «Un lien extrêmement puissant unissait en effet les membres d'un même clan et les séparait des autres, dont les vertus étaient littéralement inassimilables: "Les ancêtres n'agréent pas les sacrifices de ceux qui ne sont pas de leur espèce, les gens ne doivent pas sacrifier à ceux qui ne sont pas de leur lignage (shen bu xin fei lei min bu sifei zu)" "19. . Cette phrase du Zuozhuan trouve un écho dans la formule du Lunyu: «Sacrifier à des ancêtres qui ne sont pas les siens est pure flagornerie (fei qi gui er ji zhi, chan ye) ${ }^{20}$... » Pour cette raison, l'adoption d'un enfant autre que le fils d'un frère est strictement interdite par les canons rituels : «Un étranger au lignage sera donc considéré comme un traître potentiel, d'autant plus que les liens qui l'unissent au porteur d'un autre nom sont artificiels et peuvent être à tout moment dénoués ${ }^{21}$. $\gg$ Le seul mode d'adoption reconnu selon ce modèle classique est le don par un homme d'un de ses fils à un frère. L'idéal abstrait qu'on pourrait y voir logiquement serait de se répartir les enfants d'un même lignage entre frères. Aucune stratégie visant à greffer des enfants sur une souche stérile n'est officiellement tolérée dans cette société si farouchement attachée a son identité lignagère puisque seul un descendant du même nom, et par conséquent des «mêmes os », peut rendre le culte dû aux ancêtres de la lignée de son père. Et pourtant, ces règles canoniques sont transgressées et de telles adoptions ont bien lieu, révélant la part réellement prise par les femmes dans ce domaine ${ }^{22}$.

\section{De la greffe: le rhizome maternel}

De fait, la réalité, telle qu'on l'observe aujourd'hui, donne à voir des situations beaucoup moins orthodoxes que celles qui étaient prônées par les canons

19. Zuozhuan, Xigong, 10.3: 1801, cité par Lauwaert (1991): 1.

20. Lипуи: 2463, cité par Lauwaert (1991): 1.

21. Lauwaert (1990): 59. Les étrangers au lignage ne peuvent être adoptés que par un manquement aux rites. Comme le fait la guêpe avec les «fils de la chenille» (mingling $z h i z i)$ : «La chenille du mûrier a des petits. La guêpe les emporte sur son dos. Enseignez et instruisez vos fils. Par leur bonté ils seront vos pareils». Shijing, ode 196: 451, cité par Lauwaert (1991): 14.

22. Voir notamment Ebrey (1993): 246-249, à propos de 1'adoption d'enfants apparentés par le biais des femmes. 
antiques. Un axe féminin «antigénéalogique», qui évoque plutôt la structure d'un rhizome, s'articule à l'arborescence du patrilignage ${ }^{23}$. Si la structure en arbre est filiation, le rhizome est alliance. Il donne lieu à une carte à entrées multiples. Il croît et se multiplie, il déborde tout autant les structures classiques de l'alliance que celles de la filiation. Ainsi, les femmes créent des réseaux spécifiques qui mettent en place une forme de transmission au féminin où elles manipulent, de fait, les enfants des patrilignages. Des «échanges» d'enfants ont lieu entre «sœurs jurées» (jiebai jiemei). Il s'agit d'une forme de parenté rituelle établie entre deux femmes, amies mais non pas parentes. De tels échanges conduisent parfois à un retour sous forme de mariage entre les enfants. Dans ces cas, cette forme d'adoption peut être assimilée à une forme de stratégie matrimoniale liée à la circulation d'enfants ${ }^{24}$. C'est l'une des figures du rhizome qui contrevient symboliquement et réellement à l'interdiction faite aux femmes d'adopter et d'introduire un étranger dans les lignées patrilinéaires sauf à se conformer aux règles rituelles strictes, auquel cas la mère n'est adoptive que par l'intermédiaire du père.

C'est bien ce que les rituels donnent à voir sur un plan symbolique en mettant en scène cette sorte de «terre féminine», de pépinière céleste, cultivable à volonté. De fait, le fantasme au sujet de cette complicité féminine quant à la reproduction et à la manipulation de l'essence lignagère vaut la peine de s'y attarder. C'est ce qui donne lieu au rituel de «cultiver les Fleurs» (zaihua) toujours mis en pratique de nos jours ${ }^{25}$. Véritable jardinage contrevenant à l'ordre canonique, ce rituel se charge de toutes sortes de «greffes» d'une femme fertile à l'autre stérile ou en mal d'enfant. Par ce biais, une femme peut «emprunter» un «bouton de fleur», c'est-à-dire un embryon, à la «Fleur» céleste d'une autre femme pour lui donner naissance. Au contraire, dans d'autres rituels, on conjurera une certaine divinité ou un démon de ne pas dérober l'âme d'un enfant au profit d'une autre femme qui le mettrait au monde à sa place. C'est l'objet d'une séquence du rituel de «traverser les passes» (guoguan) qui consiste à rappeler l'âme de l'enfant afin de la réinsérer dans le patrilignage d'où elle allait être détournée ${ }^{26}$.

23. J'emprunte cette image à Deleuze et Guattari (1980): 9-38.

24. Baptandier, (2006), Bray (1997): 357, A. Wolf (1980) et Waltner (1990) : 48-80, donnent de semblables informations. À propos de ces problématiques de la circulation d'enfants, de l'adoption, du fosterage et de l'alliance, voir notamment Lallemand (1993), Leblic (dir.) (2004) et Massard (1988).

25. Berthier (Baptandier) (1988). Baptandier (1996a) et (2008).

26. Baptandier (1996a) et Baptandier (2008). Les femmes, de la sorte, tissent les générations qui les traversent pour, comme on le dit aussi, «accroître la descendance» (guangsi) en «plantant des descendants» (zhongzi). On parle de diviser, partager les embryons (fentai) 


\section{De la parole séminale du père : la mère est une épouse}

Selon les canons confucéens de l'Antiquité chinoise, une femme ne devient mère que par la vertu de la parole d'un homme qui, reconnaissant son enfant comme un fils, le nomme ${ }^{27}$. Ici, ce n'est pas la mère qui est «certaine» mais bien, explicitement, le nom du père quelle que soit la mère, génitrice ou mère adoptive. Malgré l'évidence des sens, la mère est ici déjà suspecte, troublante. Selon ces mêmes canons orthodoxes, on considère que les femmes n'ont « rien à transmettre », ni nom, ni os, ni lien ancestral, car elles ne peuvent pas reproduire l'identique: l'essence de leur propre lignage, celui de leur père.

Cette représentation de la maternité vient ici comme un déni de ce qui est pourtant la singularité propre des femmes: elles seules peuvent donner naissance au «même» (à d'autres femmes) et à l'autre sexe (à des hommes) ${ }^{28}$. Tout simplement, d'après le Classique des rites (Liji), «les épouses du père sont des mères », ce qui pose ici, d'une manière singulière, la question du féminin et de la maternité. En ce sens, la mère biologique n'est pas privilégiée parmi les mères classificatoires. La parole du père, son ordre font d'une femme la mère d'un enfant. Il y a donc une grande similitude entre maternité et adoption. La maternité se situerait du côté de la culture, du social, de l'artificiel, et non pas du naturel.

On pense ici à ce que Françoise Héritier nomme la «parole séminale du père » : «L'appartenance au groupe et l'insertion dans la lignée passent par la volonté, la reconnaissance publique, l'attribution du nom, donc par une parole, pour ainsi dire séminale, qui assure l'incorporation sociale de l'enfant, vis-à-vis tant des morts que des vivants ${ }^{29}$.»

Le rôle d'une mère ainsi définie est de se conformer à son devoir de piété filiale en offrant au lignage d'un époux un fils à son image. Il permettra à cet homme de remplir son propre devoir d'instituer son père en ancêtre. En somme, les femmes «transforment» ce que les hommes «créent». Ce sont les deux éléments du verbe «façonner», zaohua, littéralement «fabriquer et transformer», dont l'un est masculin et l'autre féminin. Comme le remarque Stein, c'est cela, la création: "Tsao (zao), "fabriquer" et houa (hua), "transformer" sont équivalents de tseu-jan (ziran), "création spontanée” ou Tao (Dao). On y voit l'activité de yin et yang, ciel et terre. Dans le domaine sexuel, tsao (zao) est propre à

comme on dirait «éclaircir un semis » ou « repiquer du riz» (fenyang), expressions dont on sait le symbolisme: l'essence, l'enfant. Furth (1995): 162.

27. De fait, dans le mariage régulier, l'épouse principale du père est la mère légitime, dimu. Elle est, en droit, la mère des enfants conçus par toutes les épouses de son mari, sans être pour autant l'égale du père. Voir Lauwaert (1991): 127.

28. Sur ce point, Héritier (1996) a envisagé une nouvelle théorie structuraliste.

29. Héritier (1985): 13. Voir également Lauwaert (1991): 126-143. 
l'homme et houa (hua) à la femme ${ }^{30}$.» Cette transformation est représentée par une image, celle des «fleurs» (hua), homophone de «métamorphose» (hua), les deux termes s'écrivant avec une nuance de différence, le marqueur sémantique des plantes. C'est cette faculté qui rend les femmes éminemment suspectes aux yeux du lignage paternel.

Par ailleurs, les femmes «nourrissent» (yang). Leur lait est de même nature que le sang qui est leur part dans la conception de l'enfant. Cela occasionne d'ailleurs un devoir particulier des fils dans le deuil qu'ils portent pour leur mère décédée: la «dette du lait» et le rituel du «bol de sang ${ }^{31}$.

\section{De l'identique et de la différence : parents de l'os et de la chair}

Dans les lignées patrilinéaires, depuis les temps les plus anciens jusqu'à aujourd'hui, pères et fils sont considérés comme un seul et même corps. Le nom qui matérialise cette identité se transmet de génération en génération, et avec lui le culte des ancêtres. Dans ce sens, on dit que les hommes transmettent l'identique : «Les Rituels nous apprennent que le père et le fils ne forment qu'une même chair, et encore de nos jours on considère comme le meilleur des remèdes de donner de sa chair à manger à un parent proche frappé d'une maladie incurable ${ }^{32}$.» Cette coutume est prônée aussi bien par les règles confucéennes de piété filiale, sous le nom de gegu, littéralement «se couper un membre», que par le bouddhisme, gegu, «se couper un os». C'est bien ce que l'on faisait encore récemment en cas de maladie grave d'un parent, et ce que l'on retrouve aussi couramment évoqué dans certains mythes décrivant ce sacrifice de soi, le seul autorisé selon les règles de la piété filiale ${ }^{33}$. À l'inverse, une manière certaine de détruire une lignée en châtiment d'un crime de l'un de ses membres, sans mettre à mort l'ensemble des parents, consistait à ouvrir les tombes et à sortir, en les dispersant, les ossements des parents défunts. C'est l'une des mesures qui, pour être jugée «normale» selon le code des châtiments, peut paraître néanmoins extrême, venant s'ajouter à celle, mise en pratique, de l'évacuation de la côte de la Chine qui avait été proposée en 1657 par un transfuge de l'armée de Zheng Chenggong (Koxinga), Huang Wu, pour mettre fin à la lignée de celui-ci et le conduire à sa perte ${ }^{34}$. À l'heure de la

30. Stein (1987): 76 , note 168 .

31. Seaman (1981, rééd. 1987): 381-397.

32. Lauwaert (1991) : 109, citant le Yili, «Sangfu» 3: 1105, qui parle plus précisément d'«un seul corps» (yiti).

33. Voir Yü (2001). Voir aussi Berthier (1988) et Baptandier (2008), à propos des mythes de Linshui furen qui aurait elle-même pratiqué le gegu en faveur de ses parents.

34. Voir Baptandier (1996b) : 108; Hsieh-Kuo-ching (1932), traduction anglaise par Hsieh (1932): 559-596. Voir aussi Gernet (1972): 410, et Imbault-Huart (1893): 53. 
mort, en effet, on accorde une importance capitale à l'intégrité du corps pour le restituer au «capital» ancestral auquel il appartient.

\section{Du destin de «tuer son père» et de son détournement}

On peut à présent se poser la question de savoir quel est le discours symbolique porté par la société elle-même sur ce contexte sociologique, canonique et rituel que nous venons de décrire. Nous pouvons nous en faire une idée par le biais de la conception chinoise du destin personnel. La pensée analogique de la Chine nous tend ici un texte à déchiffrer concernant ces sujets (la parenté, la relation au père) et leur inscription dans l'ensemble des représentations de l'univers.

\section{Du destin : les stigmates de la naissance}

Tout être vivant est identifié par huit termes - huit caractères ou nombres qui qualifient l'année, le mois, le jour et l'heure de sa naissance ${ }^{35}$. Les Chinois connaissent trois sortes de nombres: la série décimale et les séries dénaire et duodénaire. Les deux dernières sont constituées de symboles, d'images. Elles établissent des correspondances entre la nature et l'univers des humains, entre les lieux, les espaces, les qualités, mais aussi les parties du corps, les orients, les couleurs, les sensations, les saveurs, les vertus qu'elles représentent. Elles sont aussi des signes calendaires: elles qualifient les jours, les mois, les heures, les années. Elles sont bien sûr des repères topographiques: chaque parcelle d'espace peut être qualifiée, nommée de la sorte. En nombre fini (respectivement dix et douze), ces signes tissent, en se combinant, un univers clos, des cycles que constitue cet ensemble des sites du temps et des occurrences spatiales. Les dix signes de la série dénaire se nomment tiangan, «troncs, tiges célestes »; les douze signes de la série duodénaire dizhi, «rameaux terrestres». Les séries combinées de dix et de douze donnent le cycle sexagésimal composé de soixante binômes. Il faut en effet soixante étapes (c'est-à-dire dix fois six, ou douze fois cinq) pour que soient réalisées toutes les combinaisons des signes des deux séries. C'est là l'une des plus anciennes manières chinoises de compter le temps. Ces deux chiffrages sont destinés solidairement à révéler, analyser, faire apparaître la composition du total ordonné que forme l'univers. Il s'agit d'une sorte d'encryptage, dans ces nombres, de la charte du monde - y compris les humains -, de son essence ${ }^{36}$. Ces signes, tout en situant et spécifiant à la fois, établissent l'ordre du discours qui

35. Voir Baptandier (1996a).

36. À propos de ces signes cycliques, voir Granet (1934, rééd. 1950): 149-300. 
véhicule la symbiose imaginaire entre les humains et le monde. À la naissance, tout être humain est «fiché» ainsi. Il détient la carte d'identité métaphorique de son destin. L'individu est alors totalement défini. Plus précisément, le tiangan du jour de sa naissance est considéré comme son ego. Par exemple, s'il s'agit d'un jour qualifié par le tiangan «jia», l'individu sera associé à l'est, au bois, à la planète Jupiter, à la couleur verte et à la saveur aigre-acide. Son viscère dominant sera le foie, sa vertu la bienveillance.

À partir de cette symbiose individu/univers, on peut observer à l'œuvre le système des cinq éléments. Suivant les phases du yin et du yang, ils se donnent naissance ou se détruisent alternativement. On dit que l'eau donne naissance au bois, qui fait naître le feu, qui produit la terre, qui engendre le métal d'où vient l'eau. L'eau, à rebours, détruit le feu, qui vainc le métal, lequel s'oppose au bois, qui l'emporte sur la terre, qui triomphe de l'eau. Les signes cycliques qui composent le thème astrologique de l'enfant, associés à ce système des cinq éléments, sont inclus eux-mêmes dans ces cycles de naissance et de mort. Sachant que le tronc céleste du jour de naissance est l'ego de l'enfant, on pourra dès lors déterminer quelles sont les forces dominantes et les faiblesses de son thème, c'est-à-dire de sa personne, et connaître les éléments/signes cycliques qui «aident» son $e g o$, et ceux qui lui «nuisent».

Ce ne sont là que les correspondances les plus grossières, il en est de plus subtiles dont le chiffrage renvoie encore à d'autres éléments constitutifs de l'identité.

\section{De la parenté: engendrement et destruction}

Ce «chiffrage» définit en effet l'identité humaine et sociale de l'enfant, et tout d'abord dans le registre de la parenté, toujours à partir d'ego, c'est-àdire de l'élément et des correspondances qui lui sont associés. Le système des cinq éléments permet de savoir que, si ego est représenté par le signe/élément métal, c'est nécessairement la terre qui le «fait naître», tandis qu'il «vainc» le bois ( $c f$. le schéma ci-après). Or, ce discours divinatoire mêle intimement différents registres de langage : celui, cosmologique, des cinq éléments; celui, astrologique, de la qualité des bazi et de leur inscription au sein de la toile universelle de l'espace-temps; mais également, prenant au mot le symbolisme des engendrements et des destructions, celui des relations de parenté et d'alliance. Il faudrait encore ajouter à ce panorama tout ce qui concerne le corps et ses symptômes, les sentiments, les émotions. On dira par exemple que ce qui fait naître $e g o$, c'est son «empreinte», yin, qui correspond donc à sa mère (l'élément terre pour un ego métal). Ce qui l'engendre, c'est son propre «désir» (shishen), ici l'eau, qui s'oppose à sa «norme» (zhengguan), 
ici le feu, l'époux pour un ego féminin. Tandis qu'ego lui-même s'oppose à sa «richesse» (cai), ici le bois, son père (ou son épouse) qui s'oppose à son «empreinte» (yin), sa mère, ici la terre. Une telle analyse ne dispensera d'ailleurs pas d'examiner également les caractères de naissance, bazi, de la mère ou du père, en regard de ceux de l'enfant ${ }^{37}$.

Cette analyse de la situation tant cosmique que sociologique d'un individu, examinée dès sa naissance, est toujours prise très au sérieux de nos jours. On y trouve des renseignements précis sur la personnalité, les talents et les défauts de l'enfant. On cherche dès lors à «corriger» (gai), à influencer, à détourner un mauvais destin. Ces données conditionnent la dation d'un nom personnel afin de contrebalancer un déséquilibre apparent. «Trop de bois » dans la disposition des huit caractères de naissance (tiangan: jia, ou yi) induira le choix d'un nom dont les caractères comporteront, par exemple, la clé du métal, ce dont on se gardera si le thème du bois est «faible», c'est-à-dire peu présent ou en mauvaise posture relationnelle dans l'horoscope. On le voit, l'écriture joue activement son rôle dans le destin humain. Les orientations professionnelles, les tendances, les goûts personnels, les risques encourus de maladies ou d'accidents apparaîtront de la même manière.

On peut voir également dans le schéma ci-après l'expression codifiée de certaines relations canoniques. Ego s'y trouve notamment en antagonisme structurel avec son père, sa « richesse », cai (ici le bois), identifié au signe que vainc le sien propre (ici le métal). Plus spécifiquement encore, on apprendra ce qu'il en est de ses relations personnelles de parenté : se «heurte-t-il » (chong), s'oppose-t-il particulièrement violemment à son père ou à sa mère ${ }^{38}$ ? Faut-il alors le donner en adoption pour éviter un malheur ${ }^{39}$ ? On y verra encore ses

37. On effectue cette démarche dès le moment de la conception, puis à la naissance, et encore dans les moments de crises éventuelles. Au sujet de l'éducation prénatale, voir Despeux (2003) et Furth (1999).

38. Marjorie Topley (1974) a décrit le cas d'une incompatibilité entre le destin d'une mère et celui de son enfant, ce qu'elle nomme «a mother-child syndrome» et qu'elle attribue, à juste titre, à un «antagonisme cosmique». Si l'on prend en considération l'ensemble des éléments du thème de naissance, les bazi et leurs relations, on peut y trouver de la même manière une opposition au père à travers les équivalences symboliques établies entre signes cycliques, éléments et relations de parenté. Ce point est utilisé de manière très courante actuellement dans les consultations des devins. Voir Baptandier (2003).

39. On sait, par ailleurs, l'importance accordée dans une famille chinoise à la ressemblance entre l'enfant (surtout le fils) et son père: un fils doit être à l'image de son père. Un enfant infirme est une insulte faite à toute la lignée et constitue la preuve que son essence commune, jing, est pervertie. Une telle situation peut constituer une raison de donner un enfant en dehors de la famille. Certains mythes, comme par exemple l'histoire des 
possibilités d'alliance : quel(le) époux(se) choisir pour un ego métal, afin que leur relation soit harmonieuse selon les codes?

L'horoscope, les huit signes du destin d'un individu sont en somme une sorte de message mnésique indélébile, inscrit en lui de par sa position spatiotemporelle qui conditionne sa vie sociale. Et cette charte est en harmonie avec les codifications canoniques.

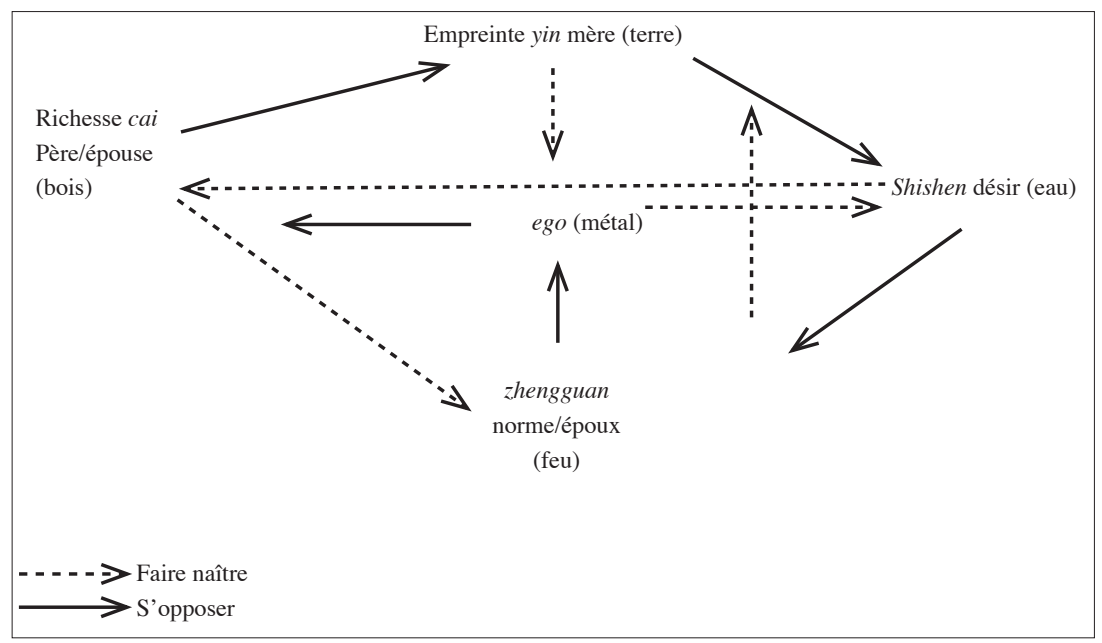

\section{Du «nœud raté» et de la lignée brisée: la «lame du yang», yangren}

Certaines personnes se trouvent dans une situation périlleuse quant à l'alliance et à la sexualité. Pourquoi cela arrive-t-il? C'est là encore un tour du destin tel qu'il se lit dans les bazi. Ce souci est dû à la conjoncture du yangren, la «lame yang ». Ce nom porte un sens condensé connoté de violence et de danger. On dit: «Si le guan (la norme) et le yin (l'empreinte) s'accordent, alors c'est le bonheur, mais s'ils s'opposent, alors c'est le yangren. Cette conjoncture est très mauvaise, on peut en mourir ${ }^{40}$.» Le texte du Sanming tonghui détaille les

Neuf Seigneurs immortels (Jiu xiangong) qui auraient vécu sous l'empereur Wu des Han (140-86 avant J.-C.), se font l'écho de cette crainte. Leur père, ne supportant pas l'image que lui renvoyaient ses fils aveugles, avait préféré les séquestrer pour les dérober à la vue des gens. Ils réussirent pourtant à s'échapper et, devenus des immortels, ils se transformèrent en carpes, symboles de longévité. On leur rend actuellement un culte au Shizhu shan (au Fujian: à Fuqing et aussi à Hanjiang), où on les sollicite pour obtenir des rêves divinatoires. Voir Baptandier (1996c).

40. D'après l'ouvrage de référence en la matière, le Sanming tonghui (ca 1550), juan III : 56-69. Selon l'ouvrage de Lin Yinghua (1979): 80-81, dans le cycle combiné des «troncs célestes » 
règles d'opposition au sein des signes cycliques dits «troncs célestes » (gan) et donne la liste des risques spécifiquement encourus chaque fois. Plusieurs classiques cités enseignent que les gens affligés d'une telle conjoncture au sein de leurs bazi auront facilement un tempérament de guerrier. Ils auront peu de fils, ou bien leur épouse, avec laquelle ils n'auront pas «d'affinité », mourra de malemort. Pour une femme, l'heure venue, son accouchement sera dangereux, ou bien encore ses deux parents mourront. Tels sont les maux les plus graves provoqués par le yangren. Ils touchent père et mère, enfants, époux et épouse, notamment lorsque celle-ci est enceinte. Le Mingli zhexue (Philosophie des principes du destin) dit encore à ce sujet : «Ceci est un châtiment (xing) exceptionnel ${ }^{41}$. Ceux qui rencontrent cette "étoile" (xing) ont un caractère violent, impatient, dévoré d'inquiétude. Les militaires ont ce destin. Ceux qui l'ont se feront nécessairement une réputation. [...] S'il s'agit d'un homme et que ses bazi sont forts, cette "lame" (ren) produira des malheurs. Les hommes qui ont le yangren, auront à coup sûr un mariage difficile. Nombre de yangren nuisent à leur femme et traumatisent leurs enfants. Quant aux femmes qui ont le yangren en relation avec le "site astrologique du mari" (fugong), elles le conduiront nécessairement à la malemort. Certains yangren pourront aussi être aveugles ou sourds-muets ».

Ces énoncés sibyllins sont limpides pour les devins. Ils évoquent par exemple yangdianxian, l'épilepsie, xian signifiant aussi la démence, la folie. Dans le discours populaire, cela s'énonce sur un plan moral: on dit par exemple qu'un tel destin survient en rétribution karmique d'un trop grand bonheur dans une vie antérieure auquel «l'amertume» $(k u)$ succède dans cette vie. Cette idée du danger encouru en raison de l'acmé de la force yang, reprend surtout le thème bien connu des métamorphoses (yi) et de la nécessité de savoir saisir le point de basculement d'un élément pour lui permettre de donner naissance harmonieusement à celui qui lui succède en évitant sa phase culminante, destructrice ${ }^{42}$.

et des «rameaux terrestres» (tiangan et dizhi), au sein du luming (le sort et ses évolutions) d'une personne, «on nomme yangren (ou ren) le rameau terrestre qui correspond au règne (diwang) du tiangan du jour de naissance. [...] Si le ren est trop prospère (wang), alors il est de nature violente et ardente et devient un souffle pervers». À propos de ce mot wang, le Shuowen jiezi dit: «C'est ce qui amène le feu à son point d'incandescence, et la prospérité à un point d'achèvement grandiose». À propos de la relation entre science calendaire et rationalisme divinatoire, voir Vandermeersch (1980) et Kalinowski (1983), (1991).

41. Huang Youde (1977): 90-91. Dans ce contexte, xing correspond à l'absence totale d'accord au sein des cinq agents, laquelle mérite «châtiment». Voir Kalinowski (1991): chap. XX.

42. Dans la charte divinatoire du Yijing, tandis que la série des figures (chaque bloc de six lignes, les hexagrammes) constitue l'élément stable du dispositif, le trait représente 


\section{De la sublimation et des remèdes empoisonnés : «corriger le destin» (gaiyun)}

\section{De l'adoption}

Lorsqu'un enfant souffre d'un déséquilibre entre les éléments représentés par les «huit caractères» de sa naissance, voire d'un déséquilibre entre ses bazi personnels et ceux d'un membre de sa famille, il suffit, dans la plupart des cas, de faire pratiquer un rituel pour transformer son destin (gaiyun), et le cas échéant pour passer la guan qui obstrue son chemin. Si les antagonismes devaient s'aggraver et si les risques s'avéraient trop importants pour l'enfant, pour sa mère ou pour son père, il faudrait alors envisager de le faire adopter. Il peut suffire, tout en gardant l'enfant dans la demeure familiale, de le donner à une divinité qui évitera, par son entremise, les «heurts» astrologiques entre le parent et l'enfant, ou bien à une autre famille dont le destin semble prospère. Il s'établit alors entre l'enfant et sa famille d'adoption un lien privilégié qui n'est pas de nature contraignante. Le remède est plus douloureux lorsqu'il marque une séparation réelle de l'enfant et de ses parents. Même si ce qui se joue dans un tel cas est plutôt de l'ordre du simulacre d'adoption et n'implique pas nécessairement une rupture avec les ancêtres, la condition d' «enfant adopté» (gan'er) est de toute façon redoutée.

Elle coupe les garçons du lien vital qui les relie à leurs ancêtres et les prive ainsi d'une part essentielle de leur identité, en particulier s'ils sont donnés comme gendres adoptés. Les filles, pour lesquelles le cas de figure est différent, ne sont pas mieux loties pour autant. Qu'elles soient données comme filles adoptives lorsque leur destin le leur permet est un moindre mal et aussi le cas le plus rare: peu de familles veulent nourrir une enfant destinée à les quitter pour se marier.

\section{De la barbarie latente: la fille est une «épouse nourrie depuis l'enfance» (tongyang $x i$ )}

Pourtant, certaines enfants, dit-on, ne peuvent demeurer comme «filles» dans leur lignage, car elles contreviennent au destin de leur père. Elles le «heurtent» (chong) et pourraient le faire mourir. Elles présentent de surcroît

l'élément individualisant et mobile. En effet, entre deux figures, seul un trait change. C'est à son niveau que s'opère la détection de l'évolution en cours et que les figures communiquent entre elles. Par la consultation relative au trait, on peut «analyser» dans le plus grand détail la logique propre à la tendance qui point, et la mettre à profit. On peut penser également à la crainte, évoquée en d'autres termes par Lewis (2006), qu'une unité poussée à son extrême n'induise la destruction. 
un risque d'antagonisme avec leur futur mari qui, faisant d'elles des épouses redoutées, les exclut du schéma habituel de l'alliance. On lira dans les bazi d'une telle enfant que «ses ancêtres n'ont rien à lui transmettre». En effet, elle «n'a pas de cai», ce qui correspond, pour une femme, au père et donc aux ancêtres. Dans un cas semblable, les filles doivent être données non pas comme enfants adoptées mais exclusivement comme «épouses nourries depuis l'enfance» (tongyang xi), et ce dès leur plus jeune âge. C'est une manière de compenser ce qui leur manque : le cai, défini comme «ce qui nourrit ma vie». Sans doute aussi ce nourrissement est-il indispensable pour détourner de leur futur époux la malemort qu'elles portent dans leur destin. Une enfant donnée très jeune, parfois à quelques mois, vivra dès lors avec son mari dans ce statu quo jusqu'à l'âge de consommer le mariage, lequel pourtant n'a aucune existence statutaire, dans le code confucéen en tout cas, ce qu'on peut comprendre. Les tongyang xi étaient généralement traitées en servantes, rudoyées et finalement obligées d'épouser celui qu'elles considéraient plutôt comme un frère. Déjà source de conflits autrefois, cette situation très fréquente occasionna maints drames. Cette pratique est désormais interdite par les lois sur le mariage et n'a donc théoriquement plus lieu dans les nouvelles générations, bien qu'on puisse encore en observer des exemples ${ }^{43}$.

Ces épouses dépréciées sont le plus souvent acceptées par des hommes trop pauvres pour conclure une alliance selon le modèle habituel, bien que cela ne se vérifie pas toujours. C'est dire que, bien évidemment, d'autres facteurs, comme notamment la pauvreté endémique en Chine, sont intervenus dans ce mode de circulation des enfants qui fut d'ailleurs, à en croire certains auteurs comme Bernard Gallin ou Arthur et Margery Wolf, l'une des formes de mariage les plus courantes à Taïwan ${ }^{44}$. Il n'en reste pas moins que cette conjoncture particulière des bazi est bien présente dans le système de pensée chinois dont elle constitue un registre du discours. Les parents d'une fille ayant ce destin pourront y remédier, conformément à la coutume, en la donnant comme tongyang xi, et ceux qui seront contraints d'abandonner leur enfant pourront avoir recours à cette figure du destin, diagnostiquée a posteriori, pour se justifier. Il demeure également que la famille d'une tongyang xi n'est pas nécessairement pauvre $^{45}$. Ainsi Parfum d'Hiver, paysanne du Fujian, n'avait quant à elle jamais

43. M. Wolf (1977) : 171-190 décrit très bien cette situation de fille adoptive. Voir aussi A. Wolf (1980), Lauwaert (1991) et (2001) et Baptandier (2003).

44. Gallin (1966), A. Wolf (1980) et M. Wolf (1968). J'ai rencontré au Fujian de nombreuses tongyang xi, souvent des femmes nées dans les années 1930-1940. Cette pratique est désormais interdite, ce qui ne donne certes pas l'assurance de son inexistence.

45. On trouve au Japon des éléments de comparaison avec cette idée que les femmes ont une place mal assurée et toujours dangereuse dans les lignées. Parfois, aussi, elles se 
pu s'expliquer pourquoi ses parents l'avaient confiée à une famille encore plus pauvre que la sienne, renonçant même de la sorte, expliquait-elle, à recevoir la parcelle de terre à laquelle ils auraient pu prétendre du fait de la politique, imminente alors, de redistribution des terres promue par Mao Zedong, pour faire d'elle «l'épouse nourrie depuis l'enfance» d'un paysan inconnu, beaucoup plus âgé qu'elle, après une dizaine d'années d'enfance vécues ensemble ${ }^{46}$. Mais elle savait toutefois que sa mère avait consulté un devin pour examiner ses bazi et $\mathrm{y}$ avait vu ce destin néfaste.

Comme toutes les femmes, une telle épouse garde son nom (xing), celui de son père. Il n'y a donc pas inceste par le nom. En revanche se crée une relation très particulière entre elle et l'essence même du lignage. Si elle arrive comme nourrisson dans la famille de son époux et si cela est possible, sa belle-mère la nourrira au sein. Ce premier don de nourriture établit une relation d'identité de nature entre les deux femmes. En effet, le lait maternel est perçu comme identique au sang menstruel, la part féminine, pensait-on, dans la conception et la formation de l'enfant. Le Bencao gangmu de Li Shizhen, cité par Furth, dit: «Breast milk: it is made of transformed yin blood (it grows due to the action of the digestive system and is maintained and supported by the action of the highway and conception channels). Before conception it appears as menses below; during pregnancy it provides nourishment for the fotus ; after birth, red changing to white, it ascends as milk ${ }^{47}$.»

Par la suite, nourrie avec le riz cultivé par son beau-père et son mari, elle est en quelque sorte assimilée à ce lignage par les os, part masculine de la conception, dont le riz est l'équivalent et la substance propre du lignage. Cette nourriture représente en somme cette transmission qui lui était interdite dans le lignage de son père. Ici donc, s'il y a un parfum d'inceste dans ce mariage, c'est plutôt par la nourriture que par le nom qu'il intervient. Plus tard - et, curieusement, comme un fils de ce lignage - la tongyang xi restera dans sa nouvelle maison pour y être épousée et ne sera plus envoyée à l'extérieur. Cette forme de

chargent de masquer le désordre qui y règne et d'y rétablir la paix par le biais d'oracles médiumniques. Voir Caillet (2001): 133-164.

46. Entre 1950 et 1953 , la réforme agraire mit en pratique le slogan «la terre à celui qui la cultive » et 300 millions de paysans pauvres accédèrent à la propriété ou augmentèrent leur parcelle. Voir Domenach (1987), I: 36. Parfum d'Hiver fut donnée en 1947-1948.

47. Furth (1986): 46, Ebrey (1993): 179, citant Chen Ziming (XIII ${ }^{\mathrm{e}}$ siècle), ouvre encore d'autres perspectives à ce sujet: «The common medical theory, in fact, was that nursing was like loosing blood and depleted a woman's strength: "In ordinary families the wives nurse their children after giving birth to them. With childbirth their vital energies (ch'i) are extremely depleted. Nursing further injures the blood. Of actions that deeply endanger life and spirit, none is greater than this" (Furen daquan liangfang, 16.2a)». 
mariage met donc également les femmes dans une situation paradoxale quant à la transmission: la tongyang xi, écartée de la maison paternelle, est absorbée par celle d'un époux qui la façonne depuis l'enfance et auquel elle donnera de la sorte une descendance également façonnée à son image. Si elle «se marie» bien et, comme cela se dit, «sort de la famille [de son père]» (chujia), elle le fait à un âge précoce et pour une raison propre à son destin particulier, celui que révèle sa situation astrologique au sein de sa généalogie.

\section{De l’accroc : la fille est une épouse métissée, « recyclée»}

En situation de diaspora, les Chinois de Polynésie ont, de nos jours, un type de mariage qui manipule les mêmes éléments en les inversant cependant. Cette situation, due sans doute à un métissage culturel, donne néanmoins à voir un exemple extrême de la logique chinoise de lignage et d'alliance, mettant à profit les pratiques d'adoption et les transferts de parenté très fréquents en Océanie, pour parvenir à ses propres buts. Un père chinois peut ne pas reconnaître sa fille nouveau-née et la donner en adoption à un lignage tahitien qui la nomme. Il lui est ensuite loisible de la «récupérer» pour l'éduquer en tant qu'épouse pour l'un des fils (cousin) du lignage chinois. Nourrie par des Tahitiens, elle est pourtant, par «nature», au plus près de la lignée de son père qui la reprend, non pas comme fille, mais comme épouse d'un autre nom. C'est ce que montre Trémon:

Mais l'importance accordée au fait d'être de père chinois, qui fait préférer les femmes demies aux femmes polynésiennes, s'explique également par la possibilité de l'alliance au sein de la communauté. C'est pourquoi le père, s'il se désintéresse de sa fille «illégitime» et la laisse dans sa famille maternelle lorsqu'il se marie avec une Chinoise, lui prêtera davantage d'attention lorsqu'elle atteindra l'adolescence. Il pourra, par son biais, nouer des alliances dans la communauté. C'est ce qui s'est passé, par exemple, pour Violette et ses sœurs, nées à Raiatea d'un père chinois et d'une mère polynésienne: selon les propres termes de Violette, leur père, au moment de s'installer à Papeete, les a «distribuées » chez des «tatis qui avaient besoin de nounous». Il les a placées dans la famille de son oncle, suivant leur âge: l'aînée, Violette (qui avait 11 ans) chez sa grand-tante, la suivante chez la belle-fille, la dernière chez la fille aînée de la bellefille. C'est aussi une façon de leur donner l'éducation chinoise qu'elles n'avaient pas eue jusque-là, puisque selon Violette, elles vivaient à Raiatea dans une «maison de Tahitiens », c'est-à-dire dans leur famille maternelle.

Ce type d'opérations n'est pas systématique. Comme nous le verrons plus loin, ce sont plus fréquemment les fils demis qui sont réintégrés de la sorte dans la famille paternelle. Ces exemples montrent toutefois que les filles demies forment des partis d'autant plus acceptables qu'on les a «récupérées » suffisamment tôt pour leur donner une éducation chinoise. Violette a par la suite épousé un Chinois, mariage arrangé entre sa grand-tante et la famille de son époux pour mettre fin au concubinage de celui-ci avec une Polynésienne... On les préfère donc aux femmes polynésiennes, 
Du meurtre symbolique du père et de l'aspect insaisissable du présent

totalement étrangères aux familles chinoises, qui ne constituent souvent que de simples aventures extraconjugales ${ }^{48}$.

\section{De la castration actée: le fils est un gendre adopté, shangmen}

Les hommes qui ont ce même destin seront souvent dotés en premier lieu d'un nom personnel, ming dépréciatif, destiné à détourner magiquement l'attention du destin comme par une sorte de wishful thinking avant d'en venir à l'issue, préjudiciable pour la lignée, de donner un fils au-dehors. Parfois le nom donné prend une coloration bisexuée: ce sera par exemple un nom personnel assez courant comme meige, littéralement «petite sœur-grand frère», attribué pour tromper un mauvais sort masculin. Et l'on peut méditer sur cette vertu de l'androgynie par le nom pour sauver un père de la violence astrologique d'un fils. C'est seulement si le destin s'acharne que l'on se résoudra à donner l'enfant soit comme fils adopté, soit comme gendre uxorilocal, ce qui est un sort redoutable ${ }^{49}$. Un tel gendre prend généralement le nom du lignage d'adoption et se trouve de la sorte coupé de la ligne de transmission de ses pères qu'il «menaçait». S'il épouse la fille de son père adoptif (comme gendre adopté), il se trouve alors de surcroît en situation d'inceste par le nom. C'est là une manière radicale de détourner le destin: rompre la lignée. On notera que les hommes sont rarement donnés enfants, mais à l'âge du mariage. Ils ne sont donc pas «nourris depuis l'enfance» par le lignage de leur épouse ${ }^{50}$.

48. Trémon (2010): 192-193. L'auteur ajoute: «Cette pratique ressemble à celle de l'achat, en Chine, des yatou, fillettes "esclaves". Si, dans le cas de Violette, cette "distribution" ne semble pas avoir donné lieu à une transaction commerciale (elle s'opère au sein de la famille étendue), nous savons, grâce à quelques témoignages, que l'achat de fillettes demies s'est pratiqué, surtout, semble-t-il, dans le but de les envoyer en Chine. Cette pratique semble avoir disparu en raison du refus qu'y opposaient les familles polynésiennes.»

49. Lauwaert (1991) et (1994) a donné une interprétation subtile de cette situation à travers, notamment, l'analyse de contes des Ming. Voir aussi, pour le Japon, Mauclaire (1993).

50. Ebrey (1993), chap. 5, donne différents exemples de mariages uxorilocaux, en particulier dans les milieux aisés de la gentry des Song, qui mettent en valeur l'intérêt pour un homme de contracter un tel mariage, dans un contexte de migration par exemple, ou pour être associé à une entreprise familiale florissante. De telles pratiques pouvaient amener les fils de familles pauvres à s'offrir comme gendres abandonnant leurs propres parents, ce qui donnait lieu parfois à des procès (p. 237). Elle souligne par ailleurs le rôle souvent pris par les femmes dans ce type d'alliance et dans un tel milieu social: «The account of this family touches on themes that recur in other references to uxorilocal marriage in Sung times. There had to be some wealth for it to work, it was linked to women's property and dowries; it was associated with women making decisions; it did 
Les fils issus d'un tel mariage appartiendront totalement, ou en partie seulement, au lignage d'adoption. Certains enfants peuvent être rendus au lignage du père géniteur pour assurer la survie de sa lignée, en particulier lorsque le gendre adopté y est l'unique fils. C'est un sort qui est vécu très douloureusement, à la fois comme une négation totale et comme une transgression majeure. Il équivaut à une véritable mutilation dans ce système de parenté où un homme se doit d'instaurer son père en ancêtre en produisant un fils pour le lignage. Le gendre uxorilocal, soupçonné de mettre un père en danger, ne peut plus porter son nom. Les enfants, quant à eux, sont tiraillés entre les deux lignages, quand bien même un accord formel aurait été passé lors de l'adoption du gendre. Si le gendre a aussi perdu le nom de son père, il n'est plus alors qu'une force de travail et de reproduction brute, sans âme. Bien des larmes coulaient des yeux de certains de ces hommes, parfois paysans habituellement peu enclins à montrer leur souffrance, au récit de leur vie. Un certain Chen avait été donné plusieurs fois : d'abord en adoption comme fils dans un autre lignage dont il avait pris le nom, puis comme gendre adopté, dans un autre lignage dont il prit aussi le nom à cette occasion. Ses enfants restèrent chez sa femme et lui-même hésitait à se nommer: quel patronyme adopter?

L'épouse du gendre adopté vit un sort également amer, malgré les apparences. À proprement parler, elle ne sort pas de sa famille pour se marier (bu chujia) puisque c'est l'époux qui adopte une résidence uxorilocale. Elle se voit alors comme demeurant une «fille» et non pas une épouse, et elle a, en somme, le destin d'une «fille de son père » à vie. Si le gendre prend le nom du père adoptif, et si les deux époux partagent le même patronyme, ils se vivent dès lors, de surcrôit, comme en situation d'inceste, de «barbarie ${ }^{51} »$. On le voit, les patrilignées ne sont pas si «rectilignes» qu'il y paraît dans les textes classiques, et bien

not always lead to a change in surname; it might be repeated in successive generations; those arguing for it claimed it was based on kinship closeness; and it might involve the blurring of boundaries between lines » (p. 239-40). Pour autant, au $\mathrm{Xx}^{\mathrm{e}}$ siècle, dans les campagnes du Fujian, la situation de gendres uxorilocaux rencontrés n'était généralement pas si avantageuse. Et comme le dit d'ailleurs Ebrey elle-même: «There seems to have been little sense that uxorilocal sons-in-law themselves could continue the descent line. Uxorilocal husbands were not equated with adoptees. What they could do was work a family's property, support the old parents, and receive shares of the property in compensation.» (p. 240). La présence sur le terrain, aujourd'hui, donne de surcroît accès à l'expression de la détresse vécue et le plus souvent à la «honte» ressentie par ces hommes de ne pouvoir tenir leur rôle de fils. À propos de la société chinoise comme société basée plus sur la honte que sur la culpabilité, voir Santangelo (1997) et (2003).

51. Ces propos me furent tenus par une femme dans une telle situation au village de Changbei, près de Gutian, au Fujian. 
Du meurtre symbolique du père et de l'aspect insaisissable du présent

des situations en chiasme se créent dans leur descendance du fait des hommes donnés à l'extérieur, ou des femmes et de leurs «greffes», rituelles ou réelles.

\section{Épilogue: de la génération de l'enfant unique et des générations alternées}

La politique de l'enfant unique fut lancée en Chine en 1979. Elle a, encore plus sûrement que les différents mouvements révolutionnaires du $\mathrm{xx}^{\mathrm{e}}$ siècle, dont c'était l'un des buts majeurs, destructuré et brouillé les repères de la parenté. Tout un pan de la parentèle traditionnelle a déjà disparu de la sorte - plus de frères ni de sœurs, déjà plus d'oncles ni de tantes (nous en sommes à la seconde génération) - et avec elle tombera bientôt dans l'oubli la terminologie sophistiquée et les termes d'adresse imposés qui l'accompagnaient. Or, savoir employer les termes appropriés pour s'adresser à une personne, fût-elle de sa parentèle ou de son environnement, est à la base même du système relationnel chinois, comme de tant d'autres. C'est là ce que les parents inculquent à leur enfant, sans indulgence aucune à l'égard des fautes commises, lesquelles impliqueraient une faute déontologique dans la manière de se situer dans la hiérarchie sociale.

Les lignées et leur culte ancestral sont mis en question par ce bouleversement radical: que faire lorsqu'on n'a, pour toujours, «qu'une fille», qui partira se marier ailleurs? Comment poursuivre le culte des ancêtres paternels ?... et, désormais, celui des ancêtres maternels, tout aussi dépourvus? Le destin est cette fois impuissant à trouver des parades et le réel prend le relais du symbolique et de l'imaginaire. Les jeunes époux en sont réduits, par deux couples de grands-parents, et non plus par un seul père, à «vouloir» un enfant, à tout prix. Plutôt un fils. Les couples bien souvent se séparent, déchirés par les injonctions inconciliables de la génération qui les précède et se les arrache, rejouant selon un scénario revisité les rivalités du moment des relevailles ${ }^{52}$. À qui donc, désormais, appartiendra l'enfant unique de ce couple moderne, pourtant toujours prisonnier des rets de la pensée traditionnelle?

Car il s'agit désormais bien d'un «couple». Chacun a choisi l'autre librement, du moins en a-t-il désormais le droit. Les femmes y regagnent-elles un «nom personnel» pour autant? Le mariage tel qu'il est conçu et littéralement «mis en scène» actuellement est bien symptomatique de cette situation: course effrénée à l'installation (l'appartement/«maison » et tout ce qui va avec demeure

52. Ce contexte de la pression constante des grands-parents impossibles à déloger est en cause (de manière pas toujours avouée) dans nombre de divorces, et l'on peut en conséquence soutenir ce paradoxe que c'est le maintien, plutôt que leur perte, desdits repères identitaires qui peut être aujourd'hui facteur de dislocation de la famille. 
bien souvent inaccessibles), théâtralisation de la cérémonie qui apparaît comme l'imaginaire d'une vie rêvée, qui se dérobe désormais. En témoignent ces albums photos qui défilent sur les écrans géants offerts à la vue de tous les convives lors du banquet de noces. Il ne s'agit généralement pas de photos de moments heureux réellement partagés par les jeunes mariés, mais bien de clichés pris en studio avec des décors et des costumes loués. En somme, il s'agit d'un jeu de rôle. Pour la photo des mariés, on y joue par exemple à «je serais Kate Middleton et tu serais William». Pour les joies des fiançailles, on montrera le sketch du «week-end à la campagne», pourtant jamais vécu. Comme si tout cela ne se concevait encore et ne pouvait se donner à voir que sur le modèle traditionnel de la «face», même si, désormais, les nouveaux partenaires de ce réseau du don et du contre-don sont moins immédiatement lisiblement établis ${ }^{53}$. La réalité de la vie quotidienne reprend ses droits dès le lendemain. Tout se passe en somme, dans cette mise en scène, comme si cette nouvelle liberté proposée à cette génération de se choisir par affinité, de s'aimer pour faire advenir son propre rêve et non plus, théoriquement, pour sceller l'alliance entre deux lignages en dépit des sentiments personnels, ne pouvait encore véritablement s'assumer dans la société actuelle. La pression exercée par les parents, dont le propre vécu fut aussi passablement pertubé par les bouleversements de l'histoire du $\mathrm{Xx}^{\mathrm{e}}$ siècle, étouffe ces femmes et ces hommes, sans leur permettre d'inventer leurs propres règles. Cette présence «traditionnelle», en ce sens que la vie sexuelle du couple parental est placée en permanence sous le regard de l'ancienne génération, a des effets infantilisants plus que jamais sensibles, et est soutenue par les références à la piété filiale. Elle joue un rôle déterminant dans des phénomènes de fuite de l'un des partenaires et de recherche d'une solution alternative, extraconjugale, porteuse de la promesse de réalisation d'une masculinité ou d'une féminité «modernes», celle d'un «couple» vraiment autonome. Nombre de couples vivent ainsi «chacun pour soi» : à chacun ses soirées, ses amis, ses tâches. Ce qui est bien... traditionnel mais prend place dans une société qui a brouillé les règles anciennes. Les sentiments, les émotions, l'érotisme sont bien cachés, plombés par le sceau de l'argent.

Est-ce pour cette raison que certains, cherchant à échapper à ces contraintes, prônent désormais le «mariage nu» (luohun) ? Un «mariage nu», c'est le fait de se marier sans aucune garantie financière, comme cela se fait habituellement. C'est le contraire d'un mariage «équipé», cette sorte de mélange entre le mariage «traditionnel» et le modèle occidental, tel qu'il est fantasmé et réinterprété, avec un appartement, une voiture, une lune de miel à l'étranger. «Sans cérémonie ou même sans bague de diamant, les jeunes couples peuvent s'unir en

53. Voir Yang (1989), (1994). 
ne dépensant que 9 yuans (moins d'un euro), soit les frais d'inscription auprès du Bureau des mariages ${ }^{54}$.»

Les filles, quant à elles, circulent toujours plus dans les réseaux de l'adoption. Les cultes propitiatoires pour obtenir l'enfant mâle tant désiré sont plus que jamais d'actualité, et bien des temples s'enrichissent grâce à ces vœux. Si on fait toujours effectuer des rituels pour que ce désir de fils soit exaucé, la science moderne est désormais en mesure d'offrir un nouveau protocole au rhizome féminin: dons d'ovules et mères porteuses se chargent parfois, en échange d'une monnaie bien réelle, de la «culture des Fleurs ${ }^{55}$.

Par ailleurs, dans ce contexte de la génération de l'enfant unique, les rôles rituels respectivement attribués aux sexes, soigneusement «tissés» dans la société traditionnelle, perdent désormais leurs repères identitaires culturels. Un seul enfant - garçon ou fille - advient à un «couple». Quel rôle aura-t-il dans sa lignée, traditionnellement patrilinéaire? Et quel profit en tirera désormais celle de sa mère, qui demeure, par définition, sans fils? On voit de plus en plus de pères «maternants», ou absents, une fois accompli le devoir de procréation. Et ceci n'est pas dû au fait que les femmes travaillent, ce qu'elles font depuis longtemps dans de nombreuses classes sociales (paysanne comme ouvrière, intellectuelle ou, nouvellement, bourgeoise), mais bien parce que les rôles traditionnellement attribués à l'un ou l'autre sexe s'estompent, par force, lorsque naît un seul enfant de manière récurrente, de génération en génération. Les filles se chargent désormais de leurs vieux parents qui n'ont pas eu de fils, ce qu'on n'attendait nullement d'elles autrefois. Que devient dans ces conditions le culte des ancêtres? Le fils d'une fille peut-il rendre sans «flagornerie» un culte à ses ancêtres maternels, dès lors qu'il ne porte pas le nom de son grandpère maternel? La fille d'un fils poursuivra-t-elle, en dépit de tout, le nom de son père? En somme, pourra-t-elle instituer son père en ancêtre, rôle dévolu jusqu'ici aux hommes, en produisant elle-même un fils? Le culte des ancêtres pourrait-il désormais «traverser» les femmes, comme le font déjà, de fait, les générations? Plus que jamais, parenté et adoption se recoupent ${ }^{56}$.

54. http://chine.aujourdhuilemonde.com/le-nouveau-chinois-le-«-mariage-nu-», consulté le 12 décembre 2011.

55. Voir notamment à titre d'exemple d'actualité l'article de Lia Le Men paru dans Aujourd'hui la Chine, «Chine: le lucratif commerce des mères porteuses », consulté le 25 novembre 2011 sur www.Aujourdhuilemonde.com/chine.

56. Ebrey (1993 : 249) remarque déjà, parlant essentiellement ici des mariages uxorilocaux dans la société des Song: «Women often played active roles in arranging to continue a family through women, but there is no evidence women were happier or better off in families that adopted these courses. Not only did women at least share the sense that they were inferior second choices, but they suffered along with men from the legal 
D'un autre côté, nombre de femmes refusent le nouveau «devoir de maternité» qui leur est fait dans le contexte de cette situation à risque - une seule chance d'avoir un fils - faisant peser sur leurs épaules non pas «la moitié du ciel», comme le voulait Mao, mais tout le poids de la nouvelle société. Les conditions économiques pèsent lourd également dans la balance : nourrir, éduquer, faire gagner le «petit empereur» ou la «petite princesse», les hisser plus haut que soi dans l'échelle sociale, ou bien les envoyer à l'étranger pour qu'ils échappent à cet impératif catégorique, aussi bien qu'à la pollution et à la crise, tout cela a un prix exorbitant ${ }^{57}$ !

Le féminin se trouve, cette fois encore, mis en question différemment. Si la mère était une épouse selon les codes, l'épouse est aujourd'hui, plus que jamais, une mère, mais la parole du père est cette fois escamotée: seuls comptent les deux couples de grands-parents qui s'emparent de l'héritier, réactualisant, d'une autre façon dans la société moderne, la prééminence de générations alternées. Le père n'est certes plus un «chef », comme le voulaient les canons de l'Antiquité, mais le grand-père paternel (ou désormais également maternel), de par cette loi de n'engendrer qu'un seul enfant, est plus que jamais un «parent» pour son petit-fils... ou désormais sa petite-fille.

«On ne peut parler du féminin que dans l'après-coup d'une séparation phallique à ce point de butée qui sépare féminin du maternel dans la difficulté du dégagement des corps », dit Liliane Gherchanoc, en conclusion de l'ouvrage intitulé Invention du féminin ${ }^{58}$. Le féminin est ici bien d'actualité, s'il ne s'esquisse que sur un horizon incertain.

weaknesses of these types of marriages. Both mothers who kept their mothers at home and widows who adopted children from their natal families would undoubtedly have wished greater guarantees that other relatives could not challenge the arrangements they had made. Women were actors to be sure, and the cumulative effect of their actions contributed over time to greater acceptance of nonstandard family forms. But that was not necessarily a comfort to individual women who found their actions legally challenged». On pourrait conclure de la même manière en ce qui concerne la situation des femmes dans la société actuelle.

57. Chicharro (2010).

58. Gherchanoc (2002). 
Du meurtre symbolique du père et de l'aspect insaisissable du présent

\section{BibLIOGRAPHIE}

Alleton Viviane (1993). Les Chinois et la passion des noms. Paris : Aubier.

BAPTANDIER Brigitte (1996a). Le rituel d'ouverture des Passes. Un concept de l'enfance. L'Homme, $\mathrm{n}^{\circ}$ 137, «Chine: facettes d'identité»: 119-142.

BAPTANDier Brigitte (1996b). Pratiques de la mémoire en Chine. Le dieu des murs et des fossés de Puxi et Hanjiang. Genèses, nº 23, «Histoire politique, histoire du politique» II : 100-124.

BAPTANDIER Brigitte (2003). Façonner la divinité en soi: à la recherche d'un lieu d'énonciation. Ethnologies, n 25 (1), «Negotiating Transcendence/Négocier la transcendance $»$ : 109-153.

BAPTANDier Brigitte (2006). Le rhizome et la perle. Penser/rêver, $\mathrm{n}^{\circ}$ 9, « La double vie des mères » : 179-187.

BAPtANDIER Brigitte (2008), Fryklund Kristina Ingrid (trad.). The Lady of Linshui. A Chinese Female Cult. Stanford: Stanford University Press.

BerthiER (BAptandier) Brigitte (1988). La Dame du bord de l'eau. Nanterre: Société d'ethnologie.

Bray Francesca (1997). Technology and Gender: Fabrics of Power in Late Imperial China. Berkeley: University of California Press.

CAILlet Laurence (2001). La sangsue et les fantômes. Petites histoires de famille au Japon. In Brigitte BAPTANDIER (dir.), De la malemort en quelques pays d'Asie. Paris: Karthala.

Cheng Anne (1985). Étude sur le confucianisme Han. Paris, Collège de France, Institut des hautes Études Chinoises.

Chicharro Gladys (2010). Le Fardeau des petits empereurs. Une génération d'enfants uniques en Chine. Nanterre: Société d'ethnologie (Haute-Asie).

Couvreur Séraphin (1950). Mémoires sur les bienséances et les cérémonies, Li Ki. Paris : E. J. Brill.

Deleuze Gilles et Guattari Félix (1980). Mille plateaux : capitalisme et schizophrénie. Paris : Éditions de Minuit.

Denton Kirk A. (1998). The Problematic of Self in Modern Chinese Litterature. Hu Feng and Lu Ling. Stanford: Stanford University Press.

Despeux Catherine (2003). Bien débuter dans la vie: l'éducation prénatale en Chine. In Christine NGuyen Tri et Catherine Despeux (dir.), Éducation et instruction en Chine, vol. I, «L'éducation élémentaire», Paris/Louvain: Peeters.

Domenach Jean-Luc et Richer Philippe (1987). La Chine, tome 1. 1949-1971. Paris: Éditions du Seuil.

DoRÉ Père Henri (1914, rééd. 1966). Recherches sur les superstitions chinoises. Taipei: Chengwen.

EBREy Buckley Patricia (1993). The Inner Quarters : Marriage and the Lives of Chinese Women in the Song Period. Berkeley: University of California.

FEng Hanyi (1937). The Chinese Kinship System, Harvard Journal of Asiatic Studies, vol. II, $\mathrm{n}^{\circ} 2$. 
Furth Charlotte (1986). Blood, Body, and Gender. Medical Images of the Female Condition in China, 1600-1850. Chinese Science, no 7: 43-66.

Furth Charlotte (1995). From Birth to Birth: The Growing Body in Chinese Medicine.

In Anne Behnke Kinney (dir.), Chinese Views of Childhood. Honolulu : University of Hawai'i Press.

GaLlin Bernard (1966). Hsin-Hsing, Taiwan: A Chinese Village in Change. Berkeley: University of California.

GeERTZ Clifford (1973). The Interpretation of Cultures. New York: Basic Books.

Gernet Jacques (1972). Le Monde chinois. Paris : Armand Colin.

GHERCHANOC Liliane (2002, rééd. 2006). L'Invention du féminin. Paris : Éditions Campagne première.

Granet Marcel (1934, rééd. 1950). La Pensée chinoise. Paris: Albin Michel.

Granet Marcel (1953). Études sociologiques sur la Chine. Paris : Presses Universitaires de France.

Granet Marcel (1959). Danses et légendes de la Chine ancienne. Paris: Presses Universitaires de France.

HARPER Donald (2001). Iatromancy, Diagnosis, and Prognosis in Early Chinese Medicine.

In Elisabeth Hsü (dir.), Innovation in Chinese Medicine. Cambridge: Cambridge University Press.

Héritier Françoise (1985). La cuisse de Jupiter. L'Homme, no 94 : 5-22.

HÉRITIER Françoise (1996). Masculin/féminin: la pensée de la différence. Paris: Odile Jacob.

Herrou Adeline (2001). La Vie entre soi. Les moines taoïstes aujourd'hui en Chine. Nanterre: Société d'ethnologie (Haute-Asie).

Hou Ching-lang (1979). The Chinese Belief in Baleful Stars. In Anna SEIDEL et Holmes Welch (dir.), Facets of Taoism. New Haven: Yale University.

HouAng Youde (1967, rééd. 1983). Mingli zhexue [Philosophie des principes du destin]. Tainan: Shenzhou shuju, Daqian shijie chubanshe.

Hsien Kuo-ching (1932). Removal of Coastal Population in Early Tsing Period. Journal of Sinological Studies, vol. II, n ${ }^{\circ}$, Peking University, 1932. Traduction anglaise par Chen Tongxie, The Chinese Social and Political Science Review, $\mathrm{n}^{\circ} 15$ : 559596.

Hsu F. Lang-Kwang (1940). Concerning the Question of Matrimonial Categories and Kinship Relationship in Ancient China. T’ien Hsia Monthly, vol. XI, n 3-4, 1940-1941 : 242-269 et 353-362.

Imbault-Huart Camille (1893). L'Île Formose : histoire et description. Paris : E. Leroux. KaLinowski Marc (1983). Les instruments astro-calendériques des Han et la méthode Liu Ren. Bulletin de l'École Française d'Extrême-Orient, t. 72 : 309-419.

KaLinowski Marc (1986). Les traités du Shuihudi et l'hémérologie chinoise à la fin des Royaumes combattants. T'oung-Pao, vol. LXXII : 175-228.

Kalinowski Marc (1991). Cosmologie et divination dans la Chine ancienne. Le compendium des cinq agents, Wuxing dayi, vie siècle. Paris: École Française d'Extrême-Orient.

LaCAn Jacques (2005). Des Noms-du-Père. Paris: Seuil, «Champs freudiens ». 
LAUWAERT Françoise (1991). Recevoir, conserver, transmettre. L'adoption dans l'histoire de la famille chinoise - aspects religieux, sociaux et juridiques. Bruxelles: Mélanges chinois et bouddhiques XXIV.

LAUWAERT Françoise (1994). La mauvaise graine. Le gendre adopté dans le conte d'imitation de la fin des Ming. Études chinoises, $\mathrm{n}^{\circ} 12$ (2) : 51-92.

LAUWAERT Françoise (2001). La mort cruelle des gens de peu: quelques cas de jurisprudence dans la Chine du XIX ${ }^{e}$ siècle. In Brigitte BAPTANDIER (dir.), De la malemort en quelques pays d'Asie. Paris: Karthala.

LeBLIC Isabelle (2004). De l'adoption: des pratiques de filiation différentes. ClermontFerrand: Presses Universitaires Blaise-Pascal.

LÉvi-Strauss Claude (1967). Les Structures élémentaires de la parenté. Paris : Mouton.

LEwIS Mark Edward (1990). Sanctioned Violence in Early China. Albany, NY: State University of New York.

LewIs Mark Edward (2006). The Construction of Space in Early China. Albany, NY: State University of New York.

Le Men Lia (22/11/2011). Chine: le lucratif commerce des mères porteuses. Aujourd'hui la Chine, consulté le 25 novembre 2011 sur www.Aujourdhuilemonde.com/chine.

Lin Yinghua (1979). Zuixin bazi tuiming zhexue [Nouvelle philosophie du destin dans les bazi]. Tainan: Dafu shuju.

Maspero Henri (1927). La Chine antique. Paris: Presses Universitaires de France.

MASSARD Josiane (1988). Engendrer ou adopter: deux visions concurrentes de la parenté chez les Malais péninsulaires. Anthropologie et sociétés, n 12 (2), «Les enfants nomades », Université de Laval: 41-62.

Mauclaire Simone (1993). L'institution de l'héritier et la pratique de nourrir le gendre dans le Japon ancien. Colloque «Adoption et Fosterage», Paris, 4 et 5 juin 1993.

Ming shi 明史 (Histoire des Ming), Pékin: Zhonghua shuju, 1973.

SANGREN Steven P. (1996). Myths, Gods, and Family Relations. In Meir SHAHAR et Robert Weller (dir.), Unruly Gods : Divinity and Society in China. Honolulu: University of Hawai'i Press.

Santangelo Paolo (1997). Le passioni nella Cina imperiale. Venise: Marsilio.

Santangelo Paolo (2003). Sentimental Education in Chinese History: an Interdisciplinary Textual Research in Ming and Qing Sources. Leyde: Brill.

SeAman Gary (1981). The Sexual Politics of Karmic Retribution. In Emily Ahern et Gates Hitl (dir.), The Anthropology of Chinese Society of Taiwan, Stanford, Stanford University Press. Rééd. 1987, Taipei: Caves Books.

STEIN Rolf A. (1987, rééd. 2001). Le Monde en petit : jardins en miniatures et habitations dans la pensée religieuse d'Extrême-Orient. Paris: Flammarion.

Topley Marjorie (1974). Cosmic Antagonism: A Mother-Child Syndrome. In Arthur P. Wolf (dir.), Religion and Ritual in Chinese Society. Stanford: Stanford University Press.

Trémon Anne-Christine (2010). Chinois en Polynésie française. Migrations, métissage, diaspora. Nanterre: Société d'ethnologie (Haute-Asie).

Turner Victor N., The Ritual Process : Structure and Anti-structure. Chicago: Aldine. 
VANDERMEERSCH Léon (1977-1980). Wangdao ou la Voie royale. Recherche sur l'esprit et les institutions de la Chine archaïque. Paris: EFEO.

Waltner Ann (1990). Getting an Heir: Adoption and the Construction of Kinship in Late Imperial China. Honolulu: University of Hawai'i Press.

WAN Minying 萬民英, Sanming tonghui 三命通會 ( ca 1550), dans Siku quanshu zhenben siji, vol. III. Taiwan: Shangwu yinshuguan.

Watson Rubie (1986). The Named and the Nameless: Gender and Person in Chinese Society. American Ethnologist, $\mathrm{n}^{\circ} 13$ (4): 619-631.

Wolf Arthur P. et HuAng Chieh-shan (1980). Marriage and Adoption in China. Stanford: Stanford University Press.

Wolf Margery (1968). The House of Lim: A Study of a Chinese Farm Family. Englewood Cliffs : Prentice Hall.

Xu Zhonglin 許仲琳 (1979). Fengshen yanyi 封神演義 (Roman de l'investiture des dieux). $\mathrm{XVI}^{\mathrm{e}}-\mathrm{XVII}{ }^{\mathrm{e}}$ siècle. Beijing: Renmin wenxue chubanshe.

YAng Mayfair Mei-Hui (1989). The Gift Economy and State Power in China. Comparative Studies in Society and History, $\mathrm{n}^{\circ} 31$ (1): 25-54.

Yang Mayfair Mei-Hui (1994). Gifts, Favors and Banquets: The Art of Social Relationships in China. Ithaca: Cornell University.

Yili 儀禮 (Traité du cérémonial). Édition Shisanjing zhushu 十三經注疏. Pékin: Zhonghua shuju, 1980.

Yü Chun-fang (2001). Kuan-yin. The Chinese Transformation of Avalokitesvara. New York: Columbia University Press.

Zuozhuan 左傳. Édition Shisanjing zhushu十三經注疏. Pékin: Zhonghua shuju, 1980.

\section{Référence en ligne :}

http://chine.aujourdhuilemonde.com/le-nouveau-chinois-le-«-mariage-nu-», consulté le 12 décembre 2011. 
Du meurtre symbolique du père et de l'aspect insaisissable du présent

\section{Glossaire}

bairi guan 百日關 la passe des Cent Jours

bazi 八字

bubaozi 不抱子 ne pas porter son fils

bu chujia 不出嫁 ne pas sortir [de sa famille pour] se marier

cai 財 richesse

Caizhu de ernümen 財主底兒女們 Enfants de riches (nouvelle de Lu Ling)

chong 沖 heurter, s'opposer à

chujia 出家 sortir des générations lignagères, entrer en religion

chujia 出嫁 sortir [de sa famille pour] se marier

daibiao 代表 représenter, incarner

dao 道 «création spontanée » (traduction de Stein)

dimu 嫡母 mère légitime

diwang 帝旺 règne

dizhi 地支 signes cycliques, rameaux terrestres

erzi 兒子 fils

fashi 法師 maître ritualiste

fei qi gui er ji zhi chan ye 非其鬼而祭之諂也 Sacrifier à des ancêtres qui ne sont pas

les siens est pure flagornerie

fentai 分胎 partager les embryons

fenyang 分秩 repiquer du riz

Fengshen yanyi 封神演義 Roman de l'Investiture des dieux

fugong 夫公 mari

fuqin 父親 père

gai 改 corriger (le destin)

gaiyun 改運 corriger le destin

gan 乾 «sec» au sens de «adopté». C'est aussi le trigramme qian, le yang, le ciel

gan'er 乾兒 enfant adopté

gegu 割骨 couper de son os [pour soigner un parent]

gegu 割股 couper de sa chair [pour soigner un parent]

guan 關 passe

guan 官 la norme

guangsi 廣嗣 accroître la postérité

guansha 關繁 le démon, «tueur» de la passe

guoguan 過關 traverser une «passe»

haizi 孩子 enfant

hongbao 紅包 enveloppe rouge (don d'argent)

Hou $\mathrm{Ji}$ 后稷

hua 化 transformation, métamorphose

hua 花 fleur

Huang $\mathrm{Wu}$ 黃梧

$\mathrm{ji}$ 雞 coq

ji 吉 bénéfique 


\section{Brigitte Baptandier}

jia 甲 l'un des dix signes cycliques (tiangan)

jiapu 家譜 registre lignager

jiashi 家詩 poème de lignage

jiebai jiemei 結拜姐妹 sœurs jurées

jing 精 essence (spermatique, lignagère)

Jiu xiangong 九仙公 les Neuf Seigneurs immortels

$\mathrm{ku}$ 苦 amertume

Liji 禮記 Classique des Rites

luan 亂 chaos

luming 祿命 le sort et ses évolutions, la chance

Lunyu 論語 Entretiens (de Confucius)

luohun 裸婚 «mariage nu»

manyue 滿月 le «mois plein», les relevailles

meige 妹哥 littéralement «petite sœur-grand frère», nom protecteur dissuasif

meiyou yuanfen 沒有缘分 n'avoir pas d'affinité astrologique

ming 名 nom personnel

mingling zhi zi 蛽蛉之子 《fils de la chenille», enfant adopté

Ming shi 明史 Histoire des Ming

Nazha santaizi 哪吒三太子 Troisième prince Nazha

pai 排 disposer, mettre en ordre rituel

qin 親 parent

ren 刃 lame

sha 憊 démon

sha 殺 tuer, massacrer

shangmen 上門 gendre adopté

shen bu xin fei lei, min bu si fei zu 神不歆非類民不祀非族 Les ancêtres n'agréent pas les sacrifices de ceux qui ne sont pas de leur espèce, les gens ne doivent pas sacrifier à ceux qui ne sont pas de leur lignage

shi 屍 cadavre

Shijing 詩經 Livre des Odes

shishen 食神 désir (dans le contexte astrologique)

Shizhu shan 石竹山 mont des Pierres et des bambous

shushu 叔叔 frère cadet du père

Taisui 太歲 la «Grande Année», cycle de 60 ans combinant en les associant les signes

cycliques (10 tiangan

et 12 dizhi)

tiangan 天干 signe cyclique, tronc céleste

tongyang xi 童養媳 épouse nourrie depuis l'enfance

waijia 外家 famille de la mère

waipo 外婆 grand-mère maternelle

wang 旺 prospère

xian 癇 démence, folie

xiantian 先天 temps précédant la naissance (inné)

xiaohai 小孩 (petit) enfant 
Du meurtre symbolique du père et de l'aspect insaisissable du présent

xing 姓 nom de lignage, du père

xing 刑 châtiment

xing 星 étoile

yang 養 nourrir

yangdianxian 陽癲痭 épilepsie

yangren 陽刃 la lame du yang

yi 易 métamorphose, mutation

yi 乙 l'un des dix signes cycliques (tiangan)

Yijing 易經 Classique des Mutations

Yili 儀禮 (Classique des) Rites et cérémonies

yin 印 empreinte

yiti 一體 un seul corps

zaihua 栽花 «cultiver les Fleurs »

zao 造 fabriquer, créer

zaohua 造化 fabriquer et transformer

zhaomu 昭穆 ordre rituel de succession des générations

zhaomutong 昭穆同 d'un même côté de la parenté

zhengguan 正官 norme (dans ce contexte astrologique)

Zheng Chenggong 鄭成功

zhongzi 種子 《planter des descendants»

ziran 自然 spontané

$\mathrm{zu}$ 祖 ancêtre

Zuozhuan 左傳 Commentaire de Zuo 
